\title{
SAVING VISION: AN INTERVENTION FOR HMOS
}

Diabetic retinopathy, like other complications of diabetes, can often be deferred or prevented by appropriate diabetes care. The Diabetes Complications Control Trial (DCCT) clearly demonstrated the positive oulcomes of strict glycemic control in preventing microvascular, retinal vascular, and other complications of diabetes. ${ }^{1}$ Annual screening for ophthalmic care for diabetes is yet another objective to be attained for early treatment, yet percentages of patients undergoing this screening are continually low This, in large part, is one reason why diabetic retinopathy is the most common cause of blindness in the U.S. among working age Americans. ${ }^{2}$

As a result of the DCCT findings, many organizations are trying to define clear standards for diabetes care.

NCQA/HEDIS accreditation standards suggest that all diabetic patients should receive an annual ophthalmologic eye examination for early prevention of diabetic retinopathy. Unfortunately, to date, these recommendations have not been consistently applied. HMOs are now faced with the task of working with their diabetic population in a more proactive fashion if more favorable outcomes are desired. This decision is made more difficult because these guidelines may increase spending for diabetes care in the short term to yield positive outcomes over the long term.

We structured a program to encourage diabetic patients within a managed care orga- nization to seek an annual ophthalmology examination. The Saving Vision program includes education for all diabetic patients and increased levels of attention for patients who need incentives or assistance in getting an annual ophthalmology examination. This initiative helps address both HEDIS and NCQA objectives as well as offers a systematic approach to targeting at-risk members of the population. As with most diabetes care interventions, early detection can significantly decrease later complications.

\section{IMPROVED CARE AND LOWERED COSTS FROM DIABETIC SCREENING -}

The Diabetic Retinopathy Study was the first major clinical trial to assess the impact of photocoagulation for diabetic retinopathy. Investigators observed a $60 \%$ decrease in risk of blindness for those patients receiving photocoagulation treatment for high-risk proliferative diabetic retinopathy. ${ }^{3}$ Other studies, such as the Early Treatment Diabetic Retinopathy Study, further demonstrated the marked effectiveness of prompt and thorough treatment for diabetic retinopathy.

Within the past few years, Javitt and colleagues 5 completed several important studies that used computer modeling and incorporated population-based epidemiologic studies and multicenter clinical trials to analyze the impact of screening on cost and sight. These studies cal- culated the annual cost savings for the federal government if screening were in place for diabetic patients. Investigators estimated the annual governmental cost for one year of blindness for patients with type II diabetes less than 65 years of age to be $\$ 14,296$. The analysis showed that screening and treatment of eye disease would generate an annual savings of $\$ 247.9$ million for the federal government-and save 53,986 person-sight years-if only $60 \%$ of the patients received treatment. ${ }^{4} \mathrm{An}$ earlier study considered type I diabetes using the same parameters. The modeling for the study showed that type I diabetes cost the federal government approximately $\$ 15,205$ per individual less than 65 years of age. However, these numbers do not include the cost of public assistance or rehabilitation. For the same group at an implementation rate of $60 \%$, the study reported the savings for the federal government of $\$ 101$ million with 47,374 person-sight years saved. 5 Both studies assumed that proliferative retinopathy, as well as diabetic macular edema, was present. The Javitt studies found substantial savings to the federal government associated with prompt treatment of retinopathy in both types of diabetes.

In contrast, a similar study by Dasbach and colleagues reported that detection and treatment of proliferative retinopathy, most specific to type I diabetes, was not cost-saving from the perspective of an $\mathrm{HMO}$ or other third-party payer. However, their analysis did not take the analytic perspective of the federal budget, nor did they consider savings associated with treatment of macular edema in the larger type II diabetic patient populations.

\section{COMPONENTS OF THE EXAMINATION}

Most recently the question has not been whether an annual examination is neces sary, but rather the logistics surrounding it. Who should perform the examination, and which diagnostic tool should be used?

Studies have suggested that most physicians do not accurately detect the presence of diabetic retinopathy. ${ }^{6}$ Providers such as ophthalmologists, diabetologists, and family practitioners commonly perform eye examinations; thus, guidelines for yearly ophthalmologic referrals for diabetic patients have been developed to help maintain standards. Managed care organizations often have difficulty in achieving these goals because of lack of compliance with the referral process by patients and physicians and maldistribution of ophthalmologists within the network.

One issue is whether the patient should undergo ophthalmoscopy or fundus photography for the diagnosis of retinopathy. The study by Peters and colleagues ${ }^{7}$ exam-

Continued on page 180 
ined the cost-effectiveness of using a nonmydriatic retinal camera in an HMO setting. The patients were all part of the diabetes program within an $\mathrm{HMO}$ and had retinal photos taken during their annual examination. The nonmydriatic retinal camera was easy to use and inexpensive as part of a general diabetes examination. If serious diabetic retinopathy were uncovered, physicians were to immediately refer the patients to an ophthalmologist for possible laser therapy. Initiatives such as this one offer good models for the process of annual ophthalmology exams.

Another important study was completed by Harris and colleagues. ${ }^{8}$ They shared that undiagnosed type II diabetes was not a benign condition, with retinopathy appearing four to seven years before diagnosis of the diabetic condition.

\section{POSITIVE RESULTS ACCRUE QUICKLY}

Some pharmacy benefit management companies are combining the medical and pharmacy daabases to define more clearly their diabetic population. For example, Diversified Pharmaceutical Services is a PBM with that capability. The goal of the Saving Vision program is to identify all diabetic patients, whether controlled by insulin therapy, oral agents, or diet. This can be accomplished by using both ICD-9 codes from medical data as well as pharmacy claims data. Presently a pilot study of Saving Vision is being conducted in an IPAmodel HMO with a low baseline rate of relerral for annual ophthalmology examinations for its diabetic population. The intervention enlists physicians, ophthalmologists, patients, and plan staff to provide increased support for better compliance.

To date, results have been very positive and exceeded the previous annual referral rate after only five months. Education and facilitation of the members, as well as the physician network, are the catalysts to motivate better compliance for the annual ophthalmology examinations. Resistant patients who do not receive screening will be enrolled in more intense care to help address their issues. Many of the barriers to care can be addressed at this point, such as clenial of asymptomatic progression, lack of physician reinforcement, inability to pay, access, and fear. Through these efforts, difficulties in the process of assuring better diabetes care to patients can be identified and addressed.

Karen DeZearn, Pharm.D.

Manager of Clinical Programs Diversified Pharmaceutical Services

2970 Clairmont Rd., Suite 690 Atlanta, GA 30329

Anne Nettles, R.N., M.S., C.D.E. Manager of Diabetes Care Management Therapeutic and Outcomes Managcment Department Diversified Pharmaceutical Services 3600 West 80 th St., Suite 700 Bloomington, MN 55431

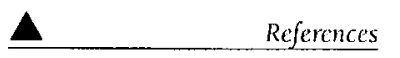

1. DCCl Research Group. The effect of intensive treatment of diabetes on the development and progression of long-term complications in insulindependent diabetes mellitus. N Engl J Med 1.993; 329: 977-86.

2. Brechner RJ, Cowie CC, Howie LJ ct al. Ophthalmic examinations among adults with diagnosed diabetes mellitus. JAMA 1993; 270: 1714-8.

3. The Diabetic Retinoparhy Research Study Group. Pholocoagulation treatment of proliferative diabetic retinopathy: clinical application of D.R.S. findings. Ophthalmology 1981; 88: 583-600.

4. Javitu JC, Ferris FL, Aiello LP et al. Preventive eye care in people with diabetes is cost-saving to the federal government. Diabetes Care 1994; 17: 909-17.

5. Javitu JC, Aiello LP, Bassi l.j et al. Detecting and treating retinopathy in patients with lype I diabetes mellius. Ophthalmology 1991; 98: 156574.

6. Sussman EJ, Tsiaras WG, Soper KA. Diagnosis of diabeic eye disease. JAMA 1982; 247: 3231-4.

7. Peters AL, Davidson MB, Zeil FH. Cost-effective screening for diabetic retinopathy using a nonmydriatic retinal camera in a prepaid healthcare setting. Diabetes Care 1993; 16 : 1193-5.

8. Harris MI, Klein R, Welborn TA, Knutman MW. Onset of NIDDM occurs at least 4-7 years before clinical diagnosis. Diabetes Care 1992; 15: 81.5-9. 